\title{
PRUEBAS ECOTOXICOLÓGICAS CON CADMIO Y CROMO USANDO POSTLARVAS DEL PEJERREY Odontesthes (Austromenidia) regia regia HILDEBRAND
}

\author{
ECOTOXICOLOGICAL. TESTS WITH CADMIUM AND CHROMIUM USING \\ POSTLARVAE OF SILVERSIDE Odontesthes (Austromenidia) regia regia \\ HILDEBRAND
}

\author{
Giovanna Vera', Jorge Tam', Víctor Vera² y Edwin Pinto'
}

\section{RESUMEN}

En el presente trabajo se determinaron las concentraciones efectivas medias (CE50\%) del cadmio $\left(\mathrm{Cd}^{* 2}\right)$ y del cromo $\left(\mathrm{Cr}^{+6}\right)$ usando postlarvas del pejerrey Odontesthes (Austromenidia) regia regia. Las postlarvas fueron expuestas a diferentes concentraciones de los metales, entre 0,142 a $1,208 \mathrm{mg} \cdot \mathrm{L}^{-1}$ de cadmio, y entre 0,53 a $33,74 \mathrm{mg}^{\mathrm{L}} \mathrm{L}^{-1}$ de cromo. Las concentraciones efectivas medias (CE50\%) obtenidas fueron de $0,648 \mathrm{mg} \cdot \mathrm{L}^{-1}$ de cadmio (a $96 \mathrm{~h}$ ) y $2,680 \mathrm{mg} \cdot \mathrm{L}^{-1}$ de cromo (a $96 \mathrm{~h}$ ). Comparativamente, el cadmio es más tóxico que el cromo y el pejerrey es más tolerante que otros organismos.

Palabras clave: Ecotoxicologia, cromo, cadmio, Odontesthes regia.

\section{ABSTRACT}

In the present paper, the mean effective concentrations (EC50\%) of cadmium ( $\mathrm{Cd}^{* 3}$ ) and chromium $\left(\mathrm{Cr}^{+6}\right)$ using postlarvae of the silverside fish Odontesthes (Austromenidia) regia regia were determined. The postlarvae were exposed to different concentrations of the metals, between 0,142 and 1.208 $\mathrm{mg} \cdot \mathrm{L}^{-1}$ of cadmium and between 0,53 and $33,74 \mathrm{mg} . \mathrm{L}^{-1}$ of chromium. The mean effective concentrations (EC50\%) obtained were $0,648 \mathrm{mg} \cdot \mathrm{L}^{-1}$ of cadmium (at $96 \mathrm{~h}$ ) and $2,68 \mathrm{mg} \cdot \mathrm{L}^{-1}$ of chromium (at 96 h). Comparatively, cadmium is more toxic than chromium, and silverside is more tolerant than other organisms.

Keywords: Ecotoxicology, chromium, cadmium, Odontesthes regia.

\section{INTRODUCCIÓN}

La ecotoxicologia ha ampliado su campo de estudio para involucrar no sólo la determinación de la relación dosis-respuesta de sustancias peligrosas a nivel de individuo, sino también los efectos a nivel de poblaciones y comunidades (Munkittrick y McCarty, 1995).

En este sentido, también es necesario ampliar el tiempo de exposición de las pruebas ecotoxicológicas para evaluar no sólo los efectos agudos, sino también los efectos crónicos

\footnotetext{
' Linea de Investigación de Ecotoxicologia Acuática, Instituto del Mar del Perú (IMARPE), Apdo: 22, Callao, Perú. 2 Unidad de Investigaciones en Acuicultura, IMARPE, Apdo. 22, Callao, Perú.
}

en especies de diferentes niveles tróficos (Stephan, 1985).

En particular, la presencia de compuestos metálicos en los ecosistemas acuáticos debido a los vertimientos de efluentes industriales ha causado graves daños a la salud humana. Los compuestos metálicos vertidos al mar, estuarios, lagos y ríos, debido a que no se biodegradan, pueden ser transferidos de un nivel trófico a otro $\boldsymbol{y}$ afectar directamente la salud humana. En Japón, por ejemplo, la contaminación por cadmio acumulado en arroz ocasionó la muerte de 230 personas en la población agricola del río Jinzu. Otro ejemplo trágico de contaminación por metales es la intoxicación por mercurio acumulado en peces y mariscos que ocasionó la muerte de $\{1\}$ per- 
sonas en la población de pescadores artesanales de la bahía de Minamata (Henry y Heinke, 1999).

En nuestro país, los estudios sobre efectos agudos y crónicos de metales en los organismos marinos son escasos, a pesar de la importancia que tiene el efectuar investigaciones para establecer criterios de calidad del agua. Los criterios de calidad acuática representan las máximas concentraciones que permiten el crecimiento y reproducción normal de los organismos, por lo que deben estar basados principalmente en pruebas de tolerancia ecotoxicológicas (Svobodova et al., 1993). Por otro lado, la información sobre toxicidad sirve de base para la evaluación del riesgo ecológico, esto es, la probabilidad de ocurrencia de efectos negativos (Gaete et al., 1996).

En vista de estos problemas, se hace necesario en el Perú desarrollar pruebas ecotoxicológicas usando organismos nativos disponibles y manejables en el laboratorio, como es el caso del pejerrey, con el fin de evaluar la toxicidad de efluentes con metales pesados tales como el cadmio.

Por tales motivos, el presente trabajo tuvo como objetivo determinar las concentraciones efectivas medias (CE50\%) de los metales cadmio y cromo, usando postlarvas del "pejerrey" Odontesthes (Austromenidia) regia regia.

\section{ANTECEDENTES}

Las pruebas de toxicidad aguda para peces han sido desarrolladas por la Administración de Alimentos y Drogas (USFDA) y la Agencia de Protección del Medio Ambiente (USEPA) de Ios Estados Unidos desde 1863 (Jones, 1964 en Humn, 1989). Estas metodologías sobre pruebas de toxicidad fueron publicadas y estandarizadas en 1960 (American Public Health Association, 1960 en Hunn, 1989), mejoradas en 1975 (Tarzwell, 1978 en Hunn, 1989) y actualizadas en 1980 (American Society for Testing and Materials,
1980 en Hunn, 1989). Estas pruebas han sido utilizadas para establecer la actividad de los quimicos en los ciclos biológicos, evaluar la toxicidad de metales y predecir el riesgo ecológico (Hunn, 1989). Entre 1974 y 1975 se firmó entre los países de la comunidad europea el convenio de París, para reglamentar los mecanismos para prevenir y proteger el Océano Atlántico y el Mar del Norte. En el marco de este convenio se ubicó al metal pesado cadmio, entre otras sustancias, en la llamada "Lista Negra", y por su alta toxicidad no se lo puede introducir en ningún ecosistema, y se debe ejecutar planes de contingencia en caso de detección de cadmio en el ambiente. Por otro lado, el metal pesado cromo ha sido catalogado en la "Lista Gris", conjuntamente con otros metales, y sólo puede ser descargado al medio acuático con extremas medidas de seguridad (Campos, 1987).

El cadmio se encuentra usualmente acopañando al zinc en aguas superficiales, pero en muy bajas concentraciones. Este metal se vierte en el ecosistema marino junto con el zinc desde fábricas de plásticos, pinturas, tubos flworescentes, tubos de televisión, baterias, industria de autos, aviones, y puede presentarse naturalmente en el petróleo crudo, gasolina, fertilizantes inorgánicos tales como fertilizantes fosfatados y lodos de aguas negras usados en tierras agrícolas. Los efluentes de minas de zine y los vertimientos municipales también son fuentes importantes de contaminación (Campos, 1987; Zweig etal. 1999). El cromo se origina en procesos de industrias de papel $y$ cartón, petroquímicos, compuestos orgánicos, soda cáustica, abonos, refinerias, metalúrgica, metales diferentes al hierro, industrias de autos y aviones, vidrios, cementos, asbestos, textiles, cueros, plantas de vapor (calderas), pigmentos, pinturas, cerámicas, fungicidas, ladrillos a prueba de fuego y catálisis. Los componentes cromados también son utilizados para control de corrosión en sistemas de caJentamiento y enfriamiento (Campos, 1987; Zweig et al., 1999). 
Tabla 1. Condiciones de las pruebas ecotoxicológicas con el metal cadmio $(+2)$ usando Odontesthes regia regia.

\begin{tabular}{|c|c|}
\hline Organismo prueba & Postlarvas de Odontesthes regia regia "pejerrey" \\
\hline Tipo de prueba & Listatica \\
\hline Duración de la prueba (horas) & 96 \\
\hline Agitación & Ausente \\
\hline Aireación & Ausente \\
\hline Agua de dilución & Agua de mar Tiltrada, estéril \\
\hline Salinidad (ups) & 35 \\
\hline Temperatura $\left({ }^{\circ} \mathrm{C}\right)$ & $16+1-1$ \\
\hline $\operatorname{Luz}(1: 0)$ & $11 \cdot 13$ \\
\hline Edad de los organismos de prueba (días) & 16 \\
\hline Número de organismos por acuario & 10 \\
\hline Número de réplicas por concentración & 3 \\
\hline Número de organismos por concentración & 30 \\
\hline Alimentación & Nauplios Arlemia sp. (a partir de las $48 \mathrm{~h}$ ) \\
\hline Limpieza de los acuarios & Ausente \\
\hline Concentraciones de prutba definitiva & 5 \\
\hline Respui ita & Mortalidad (\%) \\
\hline Criterio de aceplabilidad de la prueba & $90 \%$ o más de supervivencia en los controles. \\
\hline $\begin{array}{l}\text { El cadmio es un metal pesado que reac- } \\
\text { ciona con grupos biológicos activos (carbosil, } \\
\text { fenol, sulflidiril y otros). Larsson et al. ( } 1981 \text { ) } \\
\text { señalan que el cadmio causa variados efectos } \\
\text { subletales en peces teleósteos, como fracturas } \\
\text { y derormidad vertebral, daños testiculares, } \\
\text { desarrollo defectuoso de óvulos, reducción del }\end{array}$ & $\begin{array}{l}\text { consumo de oxígeno por los tejidos } \\
\text { branquiales, cambios patológicos en los teji- } \\
\text { dos renal e intestinal, efectos hematológicos y } \\
\text { disturbios en el metabolismo de carbohidratos. } \\
\text { Muchos de los efectos tóxicos en humanos (le- } \\
\text { siones en huesos y lesiones renales, anemia e } \\
\text { hiperglicemia) son muy parecidos a los efec- }\end{array}$ \\
\hline $\begin{array}{l}\text { Tabla } 2 \text {. Condiciones de las pruebas eco } \\
\text { Odontesthes regia regia. }\end{array}$ & cas con el metal cromo $(+6)$ usando \\
\hline Organismo prueba & Postlarvas de Odontesthes regia regia "pejerrey" \\
\hline Tipo de prueba & Estática \\
\hline Duración de la prueba (horas) & 96 \\
\hline Agitación & Auscinte \\
\hline Aireación & Ausente \\
\hline Agua de dilución & Agua de mar filtrada, estéril \\
\hline Salinidad (ups) & 35 \\
\hline Temperatura $\left({ }^{\circ} \mathrm{C}\right)$ & $16+/-1$ \\
\hline Luz (I:0) & 1113 \\
\hline Edad de los organismos de prueba (días) & 16 \\
\hline Número de organismos por acuario & 10 \\
\hline Número de réplicas por concentración & 3 \\
\hline Número de organismos por concentración & 30 \\
\hline Alimentación & Ausente \\
\hline Limpieza de los acuarios & Ausente \\
\hline Concentraciones de prueba definitiva & 7 \\
\hline Kespuesta & Mortalidad (\%) \\
\hline Criterio de aceptabilidad de la prueba & $90 \%$ o más de supervivencia en los controles. \\
\hline
\end{tabular}


Tabla 3. Concentración efectiva media (CE50\%) de cadmio (+2) usando diferentes especies acuáticas.

\begin{tabular}{|c|c|c|c|c|}
\hline Especies & Estade & $\begin{array}{c}\mathrm{CB} 508 \\
\left\langle\mathrm{ng} \cdot \mathrm{L}^{-1}\right\rangle\end{array}$ & $\begin{array}{l}\text { Tlappo do } \\
\text { oxposioion }\end{array}$ & Autor \\
\hline Poecilia reticulata & Adulteo & 0,83 & $24 \mathrm{~h}$ & Iannacone et al. (1999) \\
\hline Poecilia reticulata & Adul to & 0,72 & $48 \mathrm{~h}$ & Ianaacone et $a 1 . \quad(1999\}$ \\
\hline Crassostrea virginica & Embrion & 3.80 & $48 h$ & $\begin{array}{l}\text { Caldtasa et al. (1977) en Axiax y } \\
\text { schemiri (1982) }\end{array}$ \\
\hline Monodonta articulata & Adul to & 8,00 & $48 h$ & Axia: y Schembri (1982) \\
\hline Selenastrum capricormutum & Adulto & 0,00004 & 96 in & vymazal $(1987)$ \\
\hline Anabaena variabilis & Adul to & 0,00011 & $96 \mathrm{~h}$ & $\begin{array}{l}\text { Rach1ln et al. (1984) ex. Vymazal } \\
\text { (1987) }\end{array}$ \\
\hline Anabaena flos-aquae & Adulto & 0,00012 & $96 \mathrm{~h}$ & $\begin{array}{l}\text { Rachiln et al, (1984) en Vymazal } \\
\text { (1987) }\end{array}$ \\
\hline Nitzchia closterium & Adulto & 0,00423 & $96 h$ & $\begin{array}{l}\text { Rachiln et al. (1983b) en Vymazal } \\
\text { (1987) }\end{array}$ \\
\hline Nitzchla incerta & $A C i-2: 0$ & 0,02669 & $96 h$ & $\begin{array}{l}\text { Rachiln et al. (1983a) en Vymazal } \\
\text { (1987) }\end{array}$ \\
\hline Emerita analoga & 2 oea & 0,580 & $96 h$ & Sanchez et al. (1998) \\
\hline Chaetoceros gracilis & Adul toc & 0,595 & $96 h$ & vera et al. (en prensa) \\
\hline Odontesthes regia regia & Postiarva & 0,648 & $96 h$ & Este estudio (2002) \\
\hline Skeletonema costatua & Adulto & 1,42 & $96 h$ & Tam et a1. $\quad\langle 2000\rangle$ \\
\hline Argopecten purpuratus & Juvenil & 1,48 & $96 \mathrm{~h}$ & $\begin{array}{l}\text { Nelson et al. (1976) en Axiak y } \\
\text { Schembri (1982) }\end{array}$ \\
\hline Jordanella floridae & Tuvenil & 2,50 & $96 h$ & Spehar $(1976)$ \\
\hline Platichthys flesus & Adul to & 24,40 & $96 \mathrm{~h}$ & Larsson et al. (1981) \\
\hline Neanthes arenaceodentata & Adul to & 3,00 & $28 \mathrm{~d}$ & $\begin{array}{l}\text { Feish y Scatt (1976) en Reisbof } \\
\text { a1. (1978) }\end{array}$ \\
\hline Capitella capitata & Adul to & 7,50 & $\begin{array}{c}2 \theta d \\
\vdots\end{array}$ & $\begin{array}{l}\text { Reish y Scott (1976) en Reishet } \\
\text { a1. (1978) }\end{array}$ \\
\hline
\end{tabular}

tos del cadmio en otros mamíferos. Por otro lado, el metal cromo hexavalente es un metal potencialmente tóxico para los organismos acuáticos. carcinogénico para los mamíferos incluyendo al ser hunano (Zweig et al. 1999).

En nuestro país se han realizado escasos estudios de contaminación por metales en agua de mar (Valcárcel et al. 1974, Guillén et al. 1977, Sánchez y Hollemweguer 1991) así como en organismos marinos y en sedimentos (Echegaray et al. 1989, Guzmán 1998). Por tal motivo, existe poca información sobre cadmio y cromo en agua de mar en el Perú. En la Bahía del Callao, se han encontrado concentraciones de cadmio de $0,00158 \mathrm{mg} \cdot \mathrm{LL}^{-1} \mathrm{y}$ 0,0021 mg.L. L $^{-1}$ (Guillén et al. 1980), mientras que en la Bahía San Juanito (Ica), se han encontrado concentraciones de cromo de 0,00002 mg. $L^{-1}$ (Ministerio de Energía y Minas, com. pers.).

En curanto a las pruebas de toxicidad, podemos señalar trabajos con zinc, plomo, cobre y hierro usando Cryphiops caementarius (Bustamante 1978), con cobre usando
Argopecten purpurutus (Sánchez y Tupayachi 1988), con cromo usando Skeletonema costatum (Alayo y lannacone 1999), con cadmio usando Skeletonema costatum (Tam e. al. 2000), con cobre y cadmio usando Emerita analoga (Sánchez et al. 1998) y con metanol usando Mugil cephalus (Zambrano 1983).

En el presente trabajo se eligió como organismo prueba al pez atherínido Cdontesthes (Austromenidia) regia regics, debido a su facilidad de manejo en el laboratorio y a su gran importancia ecológica y económica. Esta especie se caracteriza por su cuerpo alargado y ornamentado por una banda lateral plateada (Chirichigno, 1998). Se distingue de otros pejerreyes relacionados que habitan en el Perú, por la presencia del premaxilar protrácíil y una aleta anal con 14 a 16 radios (Chirinos y Chuman, 1964). Frente a la zona del Callao, el pejerrey realiza desoves durante todo el transcurso del año. La primera madurez ocurre a los $13,5 \mathrm{~cm}$ de longitud. Los huevos de esta especie son semitransparentes, aproximadamente esféricos, y el tarnaño de los huevos 
Tabla 4. Concentración efectiva media (CE50\%) de cromo (+6) usando diferentes especies acuáticas.

\begin{tabular}{|c|c|c|c|c|}
\hline Espaciea & Satadio & $\begin{array}{l}\mathrm{CR} 50 \mathrm{~h} \\
\left(\mathrm{mg} \cdot \mathrm{L}^{-1}\right)\end{array}$ & $\begin{array}{l}\text { Tiespa de } \\
\text { exposicion. }\end{array}$ & Autor \\
\hline Molna macropa & $<24 \mathrm{~h}$ & 0,95 & $24 \mathrm{~h}$ & Iannacone ef al. (1999). \\
\hline Poecilia reticulaca & Adulto & $2,58-5,31$ & $24 \mathrm{~h}$ & Tannacone et a1. (1999) \\
\hline Brachionus calycitlozus & $<2 \mathrm{i}$ & 5,20 & $24 h$ & $\begin{array}{l}\text { Burbank y 5nell. (1994) en Iannacone et } \\
\text { a.1. (1999) }\end{array}$ \\
\hline Chironamus calligraphus & Larva & 13,65 & $24 \mathrm{~h}$ & Iannacone: et al. (1999) \\
\hline Odontesthes rogia regia & Postlarva & 14,13 & $24 \pi$ & Este estudio (2002) \\
\hline Poocilla reticulata & AduIto & 0,65 & $48 \mathrm{~h}$ & Iangacone et al. (1999) \\
\hline Daphnia magna & $<24 \mathrm{~h}$ & 0,10 & $48 \mathrm{~h}$ & $\begin{array}{l}\text { Munkittrick et: al. (1991: en lannacone } \\
\text { et. aI. (1999) }\end{array}$ \\
\hline Selenastrum capricoratum & Adultco & 0,08 & $96 \mathrm{~h}$ & $\begin{array}{l}\text { BLaise (1993) en Iannacone et al. } \\
\text { (1999) }\end{array}$ \\
\hline Skejetonena costacum & Adulto & 0,12 & $96 \mathrm{~h}$ & Alayo y Tannacone (1999) \\
\hline Thalassiosira mariae-leburiae & adul to & 0,9 & $96 \mathrm{~h}$ & Alayo y Iannacone $(1999)$ \\
\hline Isochrysis sp. & Adulto & 1,30 & $96 \mathrm{~h}$ & Alayo y tannacone (1999) \\
\hline Pavlova Iutheri & Adulto & 1,70 & $96 \mathrm{~h}$ & Alayo y Iannacone (1999) \\
\hline odontesthes regia regia & Postlarva & 2,68 & 9610 & Rote estudio (2002) \\
\hline Neanthes arenaceodentata & Adulto & $2,2-4,3$ & $96 \mathrm{~h}$ & $\begin{array}{l}\text { Oshida et al. (1976) en Reish et al. } \\
\text { (1978) }\end{array}$ \\
\hline Salmónidos & Juvenil. & $3,3-65$ & $96 \mathrm{~h}$ & zweig et al. (1999) \\
\hline Cerlodaphnia dubia & $<24 \mathrm{~h}$ & 3,00 & $7 \mathrm{~d}$ & $\begin{array}{l}\text { Fermandez et: al. (1959) en Iannacone et } \\
\text { al. (1999) }\end{array}$ \\
\hline Neanthes arenaceodentata & Adulto & 0,55 & $28 \mathrm{~d}$ & $\begin{array}{l}\text { Reish Y Scott }(1976) \text { en Reish et } a I \text {. } \\
\text { (1978) }\end{array}$ \\
\hline Capitella capitata & Aduito & 5,00 & $28 d$ & $\begin{array}{l}\text { Reish Y Scott (1976) en Reiah et al. } \\
\text { (1978) }\end{array}$ \\
\hline
\end{tabular}

fluctúa entre 1,30 y $2,00 \mathrm{~mm}$. El huevo fertilizado sufre poca variación durante su desarroIlo; sólo el vitelo disminuye gradualmente conforme se acerca el momento de la eclosión. El embrión está enrollado normalmente dentro del huevo, que rara vez sobrepasa los $2,00 \mathrm{~mm}$ (Chirinos y Chuman, 1964). La larva libre recién nacida mide entre $5 \mathrm{~mm}-7 \mathrm{~mm}$, a los dos o tres días absorbe el vitelo y se alimenta luego principalmente de copépodos, ostrácodos, eufäusidos, anélidos, peces, así como también. de larvas de crustáceos, anélidos y huevos de peces (Livia, 1979). Esta especie se encuentra distribuida desde Punta Aguja (Perú) a Iquique (Chile) (Chirichigno, 1998).

Estudios sobre aspectos autoecológicos del pejerrey han sido realizados por Mejía et al. (1970), Villavicencio y Muck (1984) y Coayla et al. (1991); y sobre aspectos demoecológicos por Veliz e Insil (1988), Guevara-Carrasco et al. (1991) y Fupuy (1999). El desembarque nacional de Odontesthes regia regia en el segundo semestre de 1999 fue de $33856 \mathrm{~kg}$, y el mayor desembarque ocurrió en el mes de julio
(12 185 kg), seguido de los meses de agosto y setiembre. Las caletas que presentaron los mayores desembarques fueron San Andrés (11) $429 \mathrm{~kg}$ ), Morro Sama (9286 kg) e Ilo, (10 402 $\mathrm{kg}$ ) (Estrella et al., 2000).

\section{MATERIAL Y MÉTODOS}

\section{Material Biológico}

Se realizó una colecta de ovas del pez «pejerrey» Odontesthes (Austromenidia) regia regia, durante la época de desove. Se colectaron las ovas que se encontraban adheridas a las macroalgas (Gracilaria sp. y Polysiphonia sp.) mediante buceo autónomo, cerca a la isla San Lorenzo, Callao (12 $03^{\prime} 36^{\prime \prime} \mathrm{S}$ $\left.-77^{\circ} 14^{\prime} 06^{\prime \prime} \mathrm{W}\right)$. El material biológico fue transportado en baldes con agua de mar para ser acondicionado y mantenido en laboratorio hasta que eclosionara los huevos. En las pruebas ecotoxicológicas se utilizaron postlarvas de 16 dias de eclosionadas. Las pruebas se realizaron en 1996 y se repitieron en 1999 (para cadmio) y en 2001 (para cromo). 


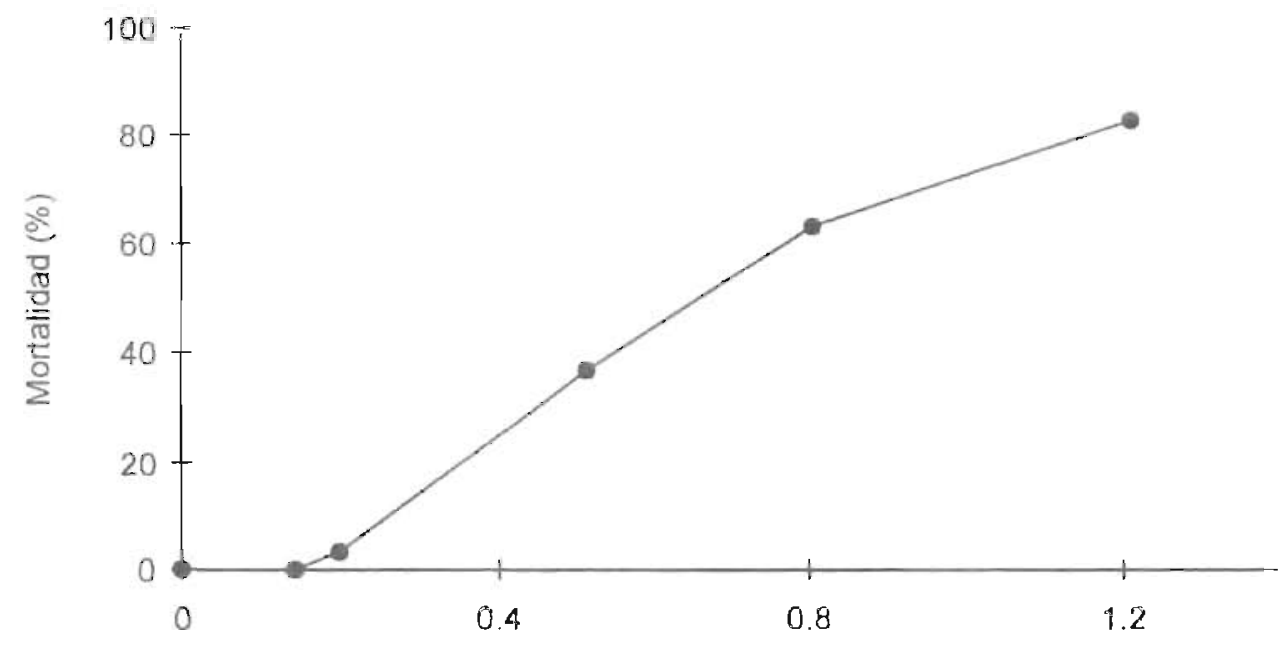

Concentración de cadmio+2 (mg.L-1)

Figura 1. Mortalidad de postlarvas de Odontesthes regia regia ai diferentes concentraciones del metal cadmio ${ }^{+2}\left(\mathrm{mg}_{\mathrm{L}} \mathrm{L}^{-1}\right)$, expuestas durante 96 horas.

\section{Métodos}

A continuación se describen los procedimientos para el acondicionamiento de las ovas en laboratorio, la separación de larvas, el mantenimiento hasta su fase de postlarva, y la ejecución de las pruebas de toxicidad, los cuales se encuentran en Sánchez y Vera (200I).

\section{Preparación de las soluciones de metales}

Se prepararon 5 diluciones de clorura de cadmio $\left(\mathrm{CdCl}_{2}\right)$ a partir de una solución stock de 949,6 $\mathrm{mg} \mathrm{L} \mathrm{L}^{-1}$ de cadmio. Se realizaron pruebas preliminares $\mathrm{o}$ 《screening test» con un rango de 0,10 a $2,00 \mathrm{mg} \mathrm{L}^{-1}$ de cadmio. De ma* nera similar, se prepararon 8 diluciones de dicromato de potasio $\left(\mathrm{K}_{2} \mathrm{Cr}_{2} \mathrm{O}_{7}\right)$ a partir de una solución stock de $2000 \mathrm{mg} \mathrm{L}^{-1}$ de cromo. Como agua de dílución se utilizó agua de mar esterilizada con luz UV. Se realizaron pruebas preliminares o «screening tests» con un rango de 1,77 a $70,70 \mathrm{mg} \mathrm{L}^{-1}$ de cromo. En base a los resultados de las pruebas preliminares se seleccionaron las concentraciones de metal para las pruebas definitivas. En el caso de cadmio, se usaron concentraciones de 0,142 , $0,199,0,512,0,802$ y $1,208 \mathrm{mg} \mathrm{L}^{-1}$. En el caso del cromo, se usaron concentraciones de 0,53 , $1,10,4,22,8,44,12,65,25,31$ y $33,74 \mathrm{mg} \mathrm{L}^{-1}$.

\section{Pruebas ecotoxicológicas}

Se realizaron pruebas con cada metal, exponiendo las postlarvas de pejerrey a las diferentes concentraciones de metales, más 1 prueba control sin metales. La prueba control comprendió solamente agua de mary las postlarvas. Se consideraron 3 réplicas por cada prueba, y se colocaron 10 postlarvas en acuarios de 1 litro, mantenidos en mesas termorregulables. Se realizaron las lecturas de sobrevivencia cada 24 horas y se extrajeron de los acuarios los organismos muertos. Como indicador de mortalidad se consideró el cese de movimientos operculares. Las pruebas fueron de tipo estático, sin aireación. Se registraron parámetros tales como temperatura $\left({ }^{\circ} \mathrm{C}\right)$ con termómetro. de mercurio, oxigeno (mg L-1) con oximetro Hanna HI 9 143, pH con un potenciómetro ESD MODEL 60 , y salinidad con un refractómetro SPARTAN A 366 ATC (ups).

\section{Análisis de datos.}

El diseño experimenta! comprendió de 6 a 8 tratamientos (5 a 7 concentraciones más I 


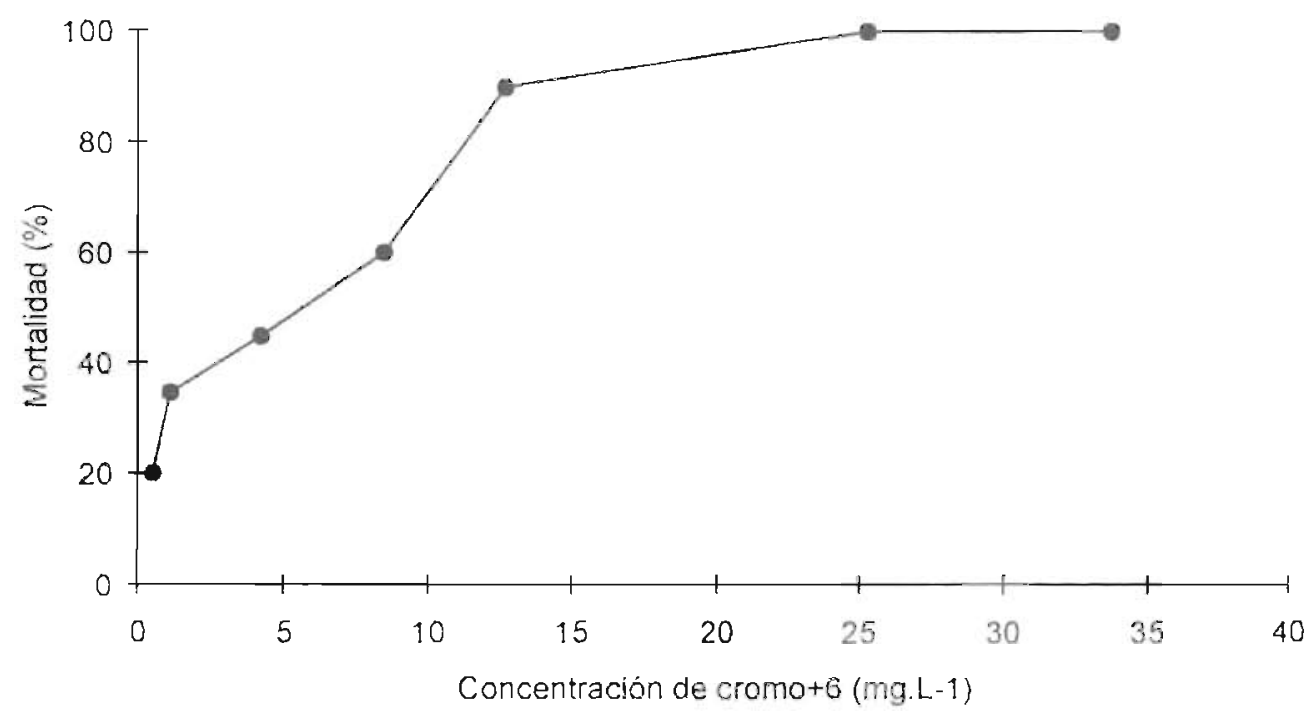

Figura 2. Mortalidad de postlarvas de Odontesthes regia regia a diferentes concentraciones del metal cromo ${ }^{+6}$ (mg. $\left.\mathrm{L}^{-1}\right)$, expuestas durante 96 horas.

control) y 3 repeticiones por cada tratamiento. La relación dosis-respuesta se obtuvo a partir de los datos de concentración como variable independiente y porcentaje de mortalidad como variable dependiente.

Se determinó la concentración efectiva media (CE50\%) utilizando el programa computacional PROBIT (Weber, 1993), que estima la concentración de cadmio y cromo correspondiente a una letalidad del 50\% de los organismos expuestos.

\section{RESULTADOS Y DISCUSIÓN}

\section{Pruebas ecotoxicológicas con cadmio}

Las condiciones de las pruebas para el metal cadmio fueron las siguientes: la temperatura se mantuvo entre $16+/-1^{\circ} \mathrm{C}$, los tenores de oxígeno variaron entre 5,8 y $6,0 \mathrm{mg} \mathrm{L}^{-1}$, el $\mathrm{pH}$ entre 8,00 y 8,11 y la salinidad fue de 35 ups (Tabla 1). La concentración efectiva media del cadmio usando postlarvas de Odontesthes (Austromenidia) regia regia fue de $0,648 \mathrm{mg} \mathrm{L}^{-1}$ a 96 horas (Fig. 1). Esta especie fue más sensible comparada con adultos de otros peces como Platichthys flesus cuya CE $50 \%$ a 96 h fue de $24,4 \mathrm{mg} . \mathrm{L}^{-1}$ (Larsson et al., 1981).

Durante el período de exposición al cadmio se realizaron observaciones del comportamien- to de los organismos. A las 24 horas todos los individuos se encontraron vivos, pero se observaron abundantes excretas en el fondo de los acuarios en las concentraciones mayores a $0,802 \mathrm{mg} \mathrm{L}^{-1}$. A. las 96 horas se observó que en los controles y en los acuarios con concentraciones menores a $0,199 \mathrm{mg} \mathrm{L}^{-1}$ los peces tenían buena natación, mientras que en los acuarios con concentraciones mayores de $0,512 \mathrm{mg} \mathrm{L}^{-1}$, los sobrevivientes estaban muy alterados y con problemas respiratorios, y se observaron movimientos operculares rápidos, a pesar de que los tenores de oxígeno disuelto estaban por encima de $5 \mathrm{mg} \mathrm{L}^{-1}$ en promedio. Los individuos muertos presentaron lesiones en la piel a manera de fisuras en la región abdominal y protrusión de las visceras. Estas observaciones coinciden con lo informado por Larsson et al. (1981), quienes señalan que los peces expuestos a metales pesados como zinc, cobre, mercurio y cadmio están propensos a sufrir alteraciones histopatológicas en los tej idos donde ocurre intercambio iónico (branquias, riñones e intestinos).

Larsson et al. (1981) comprobaron en la especie Platichthys flesus que el intercambio de iones potasio, calcio, fósforo inorgánico y 
magnesio es seriamente afectado, mas no el intercambio de los iones sodio y cloro. La reducción de calcio y potasio en el plasma puede ser responsable de las alteraciones neuromusculares tales como la hiperexcitabilidad, espasmos y contracciones de tipo tetánico (paralíticos) observados en algunos lenguados expuestos a altas concentraciones de cadmio.

\section{Pruebas ecotoxicológicas con cromo}

Las condiciones de las pruebas con el metal cromo fueron las siguientes: la temperatura se mantuvo en $16+/-1^{\circ} \mathrm{C}$, el tenor de oxígeno fue de $6.7 \mathrm{mg} \mathrm{L}^{-1}$, el $\mathrm{pH}$ entre 6,79-8,22 y la salinidad de 35 ups (Tabla 2).

La CE $50 \%$ del cromo usando postlarvas de Odontesthes regia fue de $14,13 \mathrm{mg} \mathrm{L}^{-1}$ a 24 h, 2,68 $\mathrm{mg} \mathrm{L}^{-1}$ a $96 \mathrm{~h}, 2,37 \mathrm{mg} \mathrm{L}^{-1}$ a $144 \mathrm{hy}$ $1,52 \mathrm{mg} \mathrm{L}^{-1}$ a 10 días (Fig. 2). La sensibilidad de esta especie fue muy similar a la de salmónidos juveniles cuya concentración efectiva media (CE50\%) se encuentra entre 3,3$65 \mathrm{mg} \mathrm{L}^{-1}$ (Zweig et al., 1999). Por otro lado, se observaron peces planos expuestos al cromo cubiertos de una mucosidad y con daños en el epitelio de las branquias, lo que les causaba sofocación y ahogo (Svobodova et al., 1993).

\section{Análisis comparativo}

Los criterios de calidad ambiental deben tener en consideración la tolerancia de las especies sensibles así como de las especies resistentes, a fin de proteger la mayor proporción de la comunidad acuática (Cardwell et al., 1999), En tal sentido, es necesario conocer la variabilidad y comparar la sensibilidad de especies pertenecientes a diferentes grupos taxonómicos y niveles tróficos. En la tabla 3. se presentan valores de concentración efectiva media de cadmio usando diferentes especies acuáticas, lo cual permite establecer una ordenación según la sensibilidad al cadmio de mayor a menor: Selenastrum capricornutum (microalga) mayor que Emerita analoga (crus- táceo) mayor que Chaetoceros gracilis (microalga) mayor que Odontesthes regia regia (pez) mayor que Skeleronema cosiatum (microalga) mayor que Argopecten purpuratus (molusco) mayor que Jordanella foridae (pez) mayor que. Platichlhys fesus (pez).

En la tabla 4 se presentan las concentraciones efectivas medias de cromo usando diferentes especies acuáticas, lo cula permite establecer una ordenación según la sensibilidad al cromo de mayor a menor: Selenastrum capricornuam (microaiga) mayor que Skeletonema costatum (microalga) mayor que Thalassiosira mariae-leburiae (microalga) mayor que Odontesthes regia regia (pez) mayor que Neanthes arenaceodentata (poliqueto).

En la mayoría de estos organismos acuáticos se observa que el cadmio es más tóxico que el cromo. Por otro lado, la menor sensibilidad de las postlarvas de pejerrey en comparación con otros grupos taxonómicos (crustáceos, microalgas) indica la importancia de su uso en las pruebas ecotoxicológicas como especie representante del grupo de los vertebrados.

En la Ley Generał de Aguas del Perú (El Peruano, 1969), el estándar de calidad acuática para el metal cadmio para la clase $V$ (que corresponde a. las aguas de zonas de pesca. de mariscos bivalvos) es de $0,0002 \mathrm{mg} \cdot \mathrm{L}^{\cdot 1} \mathrm{y}$ para la clase $\mathrm{V} l$ (aguas de zonas de preservación de fauna acuática y pesca recreativa o comercial) es de $0,004 . \mathrm{mg} \mathrm{L}^{-1}$. En el caso del cromo, el estándar de calidađ̃ acuática para la clase IV' (que corresponde a las aguas de zonas recreativas de contacto primario, baño o similares) es de $5 \mathrm{mg} \mathrm{L}^{-1}$, y para la clase V y VI que corresponde a las aguas de zonas de pesca de mariscos bivalvos y aguas de zonas de preservación de fauna acuática y pesca recreativa o comercial) es de $0,05 \mathrm{mg} \mathrm{L}^{-1}$. Según la USEPA (199i) e! estándar de calidad acuática en aguas marînas para el metal cadmio es de $0,043 \mathrm{mg} \mathrm{L}{ }^{-1}$ 
y para el cromo es de $0,0011 \mathrm{mg} \mathrm{L}^{-1}$. Cabe señalar que los estándares de calidad del agua en la Ley General de Aguas del Perú (El Peruano, 1969), permiten concentraciones de cromo en agua muy por encima de los 0,0125 mg.L.' de cromo, que es una concentración que produce efectos tóxicos en organismos marinos de acuerdo a Reish y Scott (1978). Por otro lado, las concentraciones de cadmio en algunos lugares del Perú se encuentran muy por encima de los estándares de calidad peruanos $\left(0,051 \mathrm{mg} \mathrm{L}^{-1}\right.$ en Callao, según Valcárcel et al., 1974). Puesto que los estándares đe calidad en ciertos casos son menos estrictos (cromo) y en otros casos son más estrictos (cadmio) en comparación con otros paises, se considera necesario revisar y actualizar la Ley Genera! de Aguas peruana vigente.

\section{CONCLUSIONES}

La concentración efectiva media (CE50\%) usando postlarvas de pejerrey con el metal cadmio y cromo fueron de 0,648 (a 96 h) y $2,68 \mathrm{mg} \cdot \mathrm{L}^{-1}$ (a $96 \mathrm{~h}$ ), lo cual indica que para esta especie el cadmio es más tóxico que el cromo.

El pejerrey presenta una tolerancia intermedia al cadmio en comparación con otras especies. La sensibilidad al cadmio de mayor a menor es: Selenasurum capricornulum (microalga) mayor que Emerita analoga (crustáceo) mayor que Chaeloceros gracilis (microalga) mayor que Odontesthes regia regia (pez) mayor que Skelelonema costatum (microalga) mayor que Argopecten purpuratus (molusco) mayor que Jordanella floridae (pez) mayor que Platichthys flesus (pez).

El pejerrey tiene una tolerancia alta al cromo en comparación con otras especies. La sensibilidad al cromo de mayor a menor es: Selenastrum capricornutum (microalga) mayor que Skeletonema cosiatum (microalga) mayor que Thalassiosira mariae-leburiae (microalga) mayor que Odontesthes regiare- gia (pez) mayor que Neanlhes arenaceodentata (poliqueto).

La tolerancia del pejerrey al cadmio y al cromo sugiere su uso como organismo representativo del grupo de los vertebrados acuáticos en las pruebas ecotoxicológicas.

\section{AGRADECIMIENTOS}

Se agradece al Sr. José Ortega por el apoyo brindado durante la colecta de material biológico y el mantenimiento de los organismos.

\section{LITERATURA CITADA}

Alayo, M. y J. lannacone. 1999. La microalga marina Sheleronema cosiatum como bioensayo alternativo para la evaluacion del cromo. VIII Congreso Latinoameicano sobre Ciencias del Mar, 17-21 de octubre. Trujillo, Perú.

Bustamante, F. 1978. Bioensayos de contaminantes metálicos hidricos y su efecto en el camarón juvenil Cryphops caementarius. Tesis Ing. Pesquera, Univ. Nac. Agraria La Molina. Lima, Perú. $91 \mathrm{pp}$.

Campos, H. 1987. Los metales pesados, su contaminación y sus efectos tóxicos. Contaminación Ambiental, Medellin. Colombia. 9(17).63-70.

Curdwell. R. D.: M. S. Brancato; J. Toll: D. DeForest and $\mathrm{L}$. Tear. 1999. Aquatic ccological risks posed by rribulytin in United States surface waters: pre-1989 to 1996 data. Env. Tox. Chem. 18:567-577.

Coayla, P: 1. Vadivia y A. Vizcarra. 1991. Contribución al comocimiento de la reproduccion del pejerrey Odontesthes regia regia, en el sur del Perú. Resúmeries del III Seminario Latinoamericano de Pesca Artesanal y lí Reurión de Desarrollo Costero Integrado, $67 \mathrm{pp}$.

Chirinos A. y E. Chuman. 1964. Notas șobre el desarrollo de huevos y larvas del pejerrey Odontesthes (Austromenidia) regia regia (Humbold1). Bol. Inst. Mar Peri 1(1):1-31.

Chirichigne. N. 1998. Clave para identificar los peces Marinos del Perú. 2." Edición. Publicación Especial Inst. Mar Perit-Callao. 427 pp.

Echegaray, M.: C. Guerin; I. Hinojosa; W. Zambranoy L. Taype, 1989. Vigilancia de la contamina. ción marina por metales pesados en áreas criticas (moluscos bivalvos como indicadores). Memorias del Simposium Internacional de los Recursos Vivos y las Pesquerias en el Pacifico 
Sudeste. Viña del Mar, 9-13 mayo 1988. CPPS. Rev. Pacifico Sur (Número Especial).

El Peruano. 1969. Ley General de Aguas. Decreto Ley N. ${ }^{\circ} 17752.24$ de julio. Perú.

L.slrella, C.; R. Guevara-Carrasco: W. Avila: I. Palacios y A. Medina. 2000. Informe estadistico de los recursos tridrobiológicos de la pesca artesanal por especies. artes, meses y culetas duranie el segundo semestre de 1999. Int lnst. Mar Perú $(151): 194$

Fupuy. J 1999. Dinámica poblacional de Odontesthes regia regia en el litoral peruano durante seliembre 1986-agosto 1996. Tesis para Licenciado en Biologia Pesquera. Univ. Pedro Ruiz. Gallo. Lambayeque. Perú. 65 pp.

(iakic. 11.: J. Silva, A Riveros. E. Soto. L. Troncoso, E. Bay-Schmidt y A. Larrain. 1996. Electo combinado y riesgo ecológico de las concentraciones de Zn, Cu y Cr presentes en el Puerto de San Vicente, Chile. Gayana Oceanol. 4:99-107.

Guevara-Carrasco. R.: C. Estrella y J. Zevallos. 1991 Crecimiento del pejerrey Odontesiltes rigia regia (Atherinidac) entre 1979-1989 en el litoral peruano. Resumenes del III Sentmario Latinoamericano de pesca artesanal y $111 \mathrm{Rc}-$ unión Desarrollo Costero Integrado. $98 \mathrm{pp}$.

Guiltén. O.: V. Asthu y R. Aquino. 1980. Contaminación marina en el Perú. Inf. Inst. Mar Perú 77:70.

(iứnén, M. 1998. Análisis comparativos del contenidu metálico en sedimentos y organismos con luerte influencia antropogénica. Resumenes de Trabajos Téenicos y Cientificos. Seminario Internacional sobre "El Estado del Medio Ambiente Marino y Costero en el Pacifico Sudeste". CPPS-PNUMA. Ecuador.

Henis. J. G. y G. W. Heinke. 1999. Ingenieria ambiental. Prentice Hall Hispanoamericana. México. $778 \mathrm{pp}$.

Hunn, J. 1989. History of acute toxicity test with fish, 1863-1987. Investigations in Fish Control. (98): 10 .

Larsson. A.; B. Bengtsson and C. Haux. 1981. Disturbed ion balance in flounder, Platichthys flesus L. exposed to sublethal levels of cadmium. Aquatic Toxicology. 1:19-35.

Livia. A. 1979. Estudios del régimen alimenticio de Odontesthes regia regia (H.) ("Pejerrey") de la caleta de Pucusana y Chorrillos. Anales cienificos UXALM, 17(I-4):53-57.

Mejía. 1.: M. Samarné y A. Pastor. 1970. Información básica de los principales peces de consumo. Inf. Insi. Mar Perú Callao. 62:1-30.

Munkittrick. K. R. and L.S. McCarty. 1995. An integrated approach to aquatic ecosystem health: top-down, bottom-up or middle-out? J. Aquat. Ecos. Health. 4:77-90.
Reish, 1) and R. Scot1. 1978. The effec of heavy metals on the survival, reproduction, development and life cycles for two species of polychaetus annelid's Alorine Pollution Bulletin 91:24-27

Sánchuz. C. y ivl. Tupayachi. 1988. Pruebas preliminarcs sobre toxicidad azuda del cobre en la conclia de abanico (Argopecten purpurahes). 191. 194 pp. Er. Salawedel. H. y A. Landa (F.ds.) Recursos y dinamica del ecosistema de afloramiento peruano Bol. En. Inst Mar P'eri Calicoo.

Sánchix, G. y Hollemweguer, E. 1991. Research and Monitoring of Marine Poltution in Peru: $1984-$ 1988. Regional Cooperation on Environmental Protection of Marine and Coastal areas of the Pacific Basin, UNEP. Regional Seas Reports and Sradies 134: 35-4.1.

Sánciuc. G.; R. Orozco y M. Jacinto. 1998. Estado de la Contaminación Marina en el Litorat Peruano en 1994 y 1995. Informe Inst. Mar Perú (36): 7-22.

Sánchez, G. y G. Vera. 200t. Manual introductorio de Ecotoxicologia Acuatica. Inf. Inst. Mar Peri. (I6I): 40 pp.

Stephan, C. E. 1985. Are the "Guidelines for deriving numerical mational water quality criteria for the protection of aquatic tife and its uses" based on sound judgements?". p. 515-526. In: Cardweli, R. D. R. Purdy and R. C. Bahner. (Eds.). Aquatic Ecotoxicology and Hazard Assessment: Siventh Symposium. Philadelphia.

Svobodova, Z, R. Lloyd: J. Machova and B. Vykusova. 1993. Water guality and nst thealh. EIFAC Technical Paper. (54):59 pp.

Tum. J.: G. Vera; E. Pinio y R. Melgar: 2000. Modelo de simulación de los efectos ecoloxicológicos del cadmio sobre el crecimiento de la microalga Skeletonema costatum (Greville) Cleve Inf. Prog. Irst. Mar Peru. 130:3-12.

U.S.E.P.A. 1991. Water Quality Criteria Summary: office of Science and Technology. Poster.

Valcárcel, G.: F Valdez y R. Venaal. 1974. Investigación sobre la contaminación de las aguns en el hitoral peruano. Min. Pesqueria del Peri. Dir Gen. Invest. Cieruitica y Tecnologica (I6):242

Velir, M. y R. Insit 1988. Variaciones mensuales y anuales de desembarque del pejerrey (Odontesthes regia regia) en el Perú entre 19.64 y 1986.69 100 p. En: Salzwedel. Hi. y A. Landa (E.ds.). Recursosy dinamica del ecosistema de a:toramiento peruano. Biol. Ext. Inst. Mar PerziCallao

Villavicencio, Z. y P. Muck. 1984. Estudio de otolitos de Odontesthes regib regia, pejerrey: Determinacion de edad. Bol. Inse Mar Perti-CaIlao. 8(3):69)-100. 
Vymazal, J 1987. Toxicity and accumulation of cadmium with respect to algae and cyanobacteria: a review. Toxicity Assessment: An international Quarterly: 2:287-415.

Whicr. (: 1. 1993. Methods for measuring the acule toxicity of effluents and receiving waters to freshwater and marine organisms. EPA600490027F. 293 pp.

7umbrano, A. W. 1983. Evaluación biológica del comportamicnto de la lisa Mugil cephalus frente al metano! utilizando metales radioquímicos. Contribucion a la Toxicologia Marina. Tesis Ing. Pesquero. Universidad Federico Villarreal. $90 \mathrm{pp}$.

¿weig R: J. Morton y M. Stewart 1999. Source water Quality for Aquaculture. A Guide for Assessment. Environmentally and Socially Sustainable Development Rural Development. 62 pp. 